\title{
SYNCHRONIZATION ANALYSIS OF INERTIAL MEMRISTIVE NEURAL NETWORKS WITH TIME-VARYING DELAYS
}

\author{
Ruoyu Wei, Jinde Cao \\ School of Mathematics, Southeast University, \\ al. Southeast University, Nanjing, China
}

Submitted: 15th January 2018; Accepted: 5th March 2018

\begin{abstract}
This paper investigates the global exponential synchronization and quasi-synchronization of inertial memristive neural networks with time-varying delays. By using a variable transmission, the original second-order system can be transformed into first-order differential system. Then, two types of drive-response systems of inertial memristive neural networks are studied, one is the system with parameter mismatch, the other is the system with matched parameters. By constructing Lyapunov functional and designing feedback controllers, several sufficient conditions are derived respectively for the synchronization of these two types of drive-response systems. Finally, corresponding simulation results are given to show the effectiveness of the proposed method derived in this paper.
\end{abstract}

Keywords: inertial, memristive, neural networks, synchronization

\section{Introduction}

The memristor is considered to be the fourth basic circuit element besides resistor, capacitor, and inductor, originally predicted by Chua in 1971 [1]. However, the first practical memristor device was realized by HP laboratory until 2008 [2]. The memristor has the property of memorizing the history of the applied voltage, which is similar to the operating principle of synapsis in human brain. Therefore, modelling the synapsis has become an important application of memristor. Recently, more and more attention has been paid to the memristor because of its potential application in next generation computers and powerful brain-link neural computers. By replacing resistor in traditional neural network with memristor, the memristive neural network is then constructed. The main character of memristive neural network is that the right-hand side of its dynamic equation switches according to state. Recently, there has been many interesting results concerning the dynamics of memristive neural networks [3-5]. In [4], the lag synchronization of memristor-based coupled neural networks with or without parameter mismatch was studied; The global exponential synchronization of drive-response memristive neural networks with heterogeneous time-varying delay was investigated in [5].

The neural network with second-order term is called inertial neural network, of which the dynamic behavior is much more complex than first-order neural networks. Physically, the second-order term represents inductance in circuit systems. There is strong biological background for the introduction of an inductance term in neural system [6-8]. In semicircular canals of some animals, the membrane of a hair cell can be implemented by equivalent circuits that contain an inductance [9-10]. Most of the results of inertial network focus on the periodic solution, bifurcation and stability [11-13]. 
Both of inertial neural network and memristive neural network have received considerable attention due to their widely application in various fields, such as pattern recognition, associative memories and learning, etc [14-17]. However, there are very few results about the neural network containing both of them. [3] discuss stability and synchronization of inertial memristive neural networks with time delays. However, the control scheme can be improved and different types of synchronization could be further considered. Due to the special physical meaning and biological background of inertial and memristor, the combination of them can model more complex dynamical behaviors in nature and extend the potential application of neural networks. Moreover, we also take time-varying delay into consideration, which may change the dynamics of systems and make it difficult to achieve synchronization. To the best of our knowledge, there are very few results on the dynamical behavior of inertial memristive neural network and this motivates our research interests.

Synchronization is an important phenomenon in nature, and has been widely applied in various fields such as chemical reactors, biological systems, information processing, secure communication, etc [18-23]. Due to the uncertainty of math model and the existence of external disturbance, it is hard to construct two identical drive-response system in practical situation, parameter mismatch is unavoidable. So it is of great importance to investigate the system with unmatched parameters. However, in this case, complete synchronization can not be achieved. Instead, we consider another type of synchronization: quasi-synchronization, which is weaker than complete synchronization, but more practical in real applications.

Motivated by above discussion, this paper investigates the synchronization problem of inertial memristive neural networks with or without parameter mismatch. To the best of our knowledge, the quasi-synchronization of inertial memristive neural networks and adaptive control method for exponential synchronization has not been fully investigated in previous papers yet. The novelties of this paper lies in the following aspects: For the first time, the adaptive control method is introduced to this model, which reduces the cost for the control. Different from previous literatures, the matrix measure method is utilized to study quasi-synchronization in this paper, which simplifies the analysis process and reduces the conservativeness of the conclusion.

The remainder of this paper is organized as follows. In Section 2, some notations and preliminaries are given. The model formulation and main results are presented in Section 3 and 4. In Section 5, three numerical examples are given to demonstrate the effectiveness of the main results. Finally, conclusions are drawn in Section 6.

Notations. Throughout this paper, $R^{n}$ denotes the n-dimensional Euclidean space. The superscript T denotes vector transposition. $R^{n \times n}$ is the set of all $n \times n$ real matrices. $I_{n}$ is the identity matrix of order n. $\operatorname{sign}(\cdot)$ is the sign function. $C^{(1)}\left([-\tau, 0], R^{n}\right)$ denotes the family of continuous functions from $[-\tau, 0]$ to $R^{n}$.

\section{Preliminaries}

Consider the model of inertial memristive neural network consisting of $n$ nodes

$$
\begin{aligned}
\frac{d^{2} s_{i}(t)}{d t^{2}}= & -d_{i} \frac{d s_{i}(t)}{d t}-c_{i} s_{i}(t)+\sum_{j=1}^{n} a_{i j}\left(s_{i}(t)\right) f_{j}\left(s_{j}(t)\right) \\
& +\sum_{j=1}^{n} b_{i j}\left(s_{i}(t)\right) f_{j}\left(s_{j}(t-\tau(t))\right)+I_{i}, \\
& i=1, \cdots, n
\end{aligned}
$$

where $s_{i}(t)$ denotes the state vector of the $i$ th neuron. $d_{i}>0, c_{i}>0$ are positive constants. The second derivative of $s_{i}(t)$ represents the inertial term. The nonlinear function $f_{i}$ stands for the activation function for the $i$ th neuron. $I_{i}$ is the external input of the system. $\tau(t)$ is the time-varying delay that satisfies $0 \leq \tau(t) \leq \tau, \dot{\tau}(t) \leq \theta<1$, where $\tau$ and $\theta$ are constants. According to the character of a memristor, the connecting weights $a_{i j}\left(s_{i}(t)\right)$ and $b_{i j}\left(s_{i}(t)\right)$ represent the non-delayed and delayed memristive synaptic connection weights, respectively, and

$$
\begin{aligned}
a_{i j}\left(s_{i}(t)\right) & =\frac{W_{a i j}\left(s_{j}(t)\right) s g n_{i j}}{C_{i}}, \\
b_{i j}\left(s_{i}(t)\right) & =\frac{W_{b i j}\left(s_{j}(t)\right) s g n_{i j}}{C_{i}}, \\
\operatorname{sgn}_{i j} & =\left\{\begin{array}{c}
1, i=j, \\
-1, i \neq j,
\end{array}\right.
\end{aligned}
$$

where $W_{a i j}\left(x_{j}(t)\right)$ and $W_{b i j}\left(x_{j}(t)\right)$ denote the memductances of memristors $M_{a i j}$ and $M_{b i j}$, respec- 
tively. $M_{a i j}$ represents the memristor between the feedback function $f_{j}\left(s_{j}(t)\right)$ and $s_{i}(t), M_{b i j}$ represents the memristor between the feedback function $f_{j}\left(s_{j}(t-\tau(t))\right)$ and $s_{i}(t)$. The capacitor $C_{i}$ is invariant while memductances of memristors $W_{a i j}\left(x_{j}(t)\right)$ and $W_{b i j}\left(x_{j}(t)\right)$ respond to change in pinched hysteresis loops. Hence, $a_{i j}\left(s_{i}(t)\right)$ and $b_{i j}\left(s_{i}(t)\right)$ will change. According to the feature of memristor and current-voltage characteristics, we consider a general mathematical model of the memristances as follows

$$
\begin{aligned}
& a_{i j}\left(s_{i}(t)\right)=\left\{\begin{array}{l}
\hat{a}_{i j},\left|s_{i}(t)\right| \leq T_{i}, \\
\breve{a}_{i j},\left|s_{i}(t)\right|>T_{i},
\end{array}\right. \\
& b_{i j}\left(s_{i}(t)\right)=\left\{\begin{array}{l}
\hat{b}_{i j},\left|s_{i}(t)\right| \leq T_{i}, \\
\breve{b}_{i j},\left|s_{i}(t)\right|>T_{i},
\end{array}\right.
\end{aligned}
$$

for $i, j=1,2, \cdots n$, where $\hat{a}_{i j}, \check{a}_{i j}, \hat{b}_{i j}, \breve{b}_{i j}$ are known constants with respect to memristances. The initial value associated with system (1) is

$s_{i}(\omega)=\Phi_{i}(\omega), \quad \frac{d s_{i}(\omega)}{d \omega}=\Psi_{i}(\omega), \quad-\tau \leq \omega \leq 0$,

where $\Phi_{i}(\omega), \Psi_{i}(\omega) \in C^{(1)}\left([-\tau, 0], R^{n}\right), \quad i=$ $1, \cdots, n$. The following assumptions are necessary for the main results of this paper.

Assumption 2.1 For all $x, y \in R, x \neq y$, the neural activation function $f_{i}(\cdot)$ satisfies

$$
\left|f_{i}(x)-f_{i}(y)\right| \leq l_{i}|x-y|, i=1, \cdots, n,
$$

where $l_{i}$ are known constants.

Assumption 2.2 There exists constants $M_{i}$ s.t. $\left|f_{i}(z)\right| \leq M_{i}, \forall z \in R, i=1 \ldots n$.

Based on the theories of set-valued maps and differential inclusions, the model (1) with initial values can be described by the following differential inclusion

$$
\begin{aligned}
\frac{d^{2} s_{i}(t)}{d t^{2}}+d_{i} \frac{d s_{i}(t)}{d t} \in & -c_{i} s_{i}(t)+\sum_{j=1}^{n} c o\left[\underline{a}_{i j}, \bar{a}_{i j}\right] f_{j}\left(s_{j}(t)\right) \\
& +\sum_{j=1}^{n} c o\left[\underline{b}_{i j}, \bar{b}_{i j}\right] f_{j}\left(s_{j}(t-\tau(t))\right) \\
& +I_{i}
\end{aligned}
$$

where $\underline{a}_{i j}=\min \left\{\hat{a}_{i j}, \check{a}_{i j}\right\}, \bar{a}_{i j}=\max \left\{\hat{a}_{i j}, \breve{a}_{i j}\right\}, \underline{b}_{i j}=$ $\min \left\{\hat{b}_{i j}, \breve{b}_{i j}\right\}, \bar{b}_{i j}=\max \left\{\hat{b}_{i j}, \breve{b}_{i j}\right\}$,

$$
\begin{gathered}
c o\left[\underline{a}_{i j}, \bar{a}_{i j}\right]=\left\{\begin{array}{c}
\hat{a}_{i j},\left|s_{i}(t)\right|<T_{i}, \\
{\left[\underline{a}_{i j}, \bar{a}_{i j}\right],\left|s_{i}(t)\right|=T_{i},} \\
\check{a}_{i j},\left|s_{i}(t)\right|>T_{i},
\end{array}\right. \\
\operatorname{co}\left[\underline{b}_{i j}, \bar{b}_{i j}\right]=\left\{\begin{array}{c}
\hat{b}_{i j},\left|s_{i}(t)\right|<T_{i}, \\
{\left[\underline{b}_{i j}, \bar{b}_{i j}\right],\left|s_{i}(t)\right|=T_{i},} \\
b_{i j},\left|s_{i}(t)\right|>T_{i},
\end{array}\right.
\end{gathered}
$$

or equivalently, for $i, j=1,2, \cdots n$, there exists $\tilde{a}_{i j}\left(s_{i}(t)\right) \in \operatorname{co}\left[\underline{a}_{i j}, \bar{a}_{i j}\right], \tilde{b}_{i j}\left(s_{i}(t)\right) \in \operatorname{co}\left[\underline{b}_{i j}, \bar{b}_{i j}\right]$ such that

$$
\begin{aligned}
\frac{d^{2} s_{i}(t)}{d t^{2}}+d_{i} \frac{d s_{i}(t)}{d t}= & -c_{i} s_{i}(t)+\sum_{j=1}^{n} \tilde{a}_{i j}\left(s_{i}(t)\right) f_{j}\left(s_{j}(t)\right) \\
& +\sum_{j=1}^{n} \tilde{b}_{i j}\left(s_{i}(t)\right) f_{j}\left(s_{j}(t-\tau(t))\right) \\
& +I_{i} .
\end{aligned}
$$

Next, by introducing the following variable transformation

$$
p_{i}(t)=s_{i}(t), q_{i}(t)=\frac{d s_{i}(t)}{d t}+s_{i}(t),
$$

system (5) can be transformed into the first-order form

$$
\left\{\begin{aligned}
\dot{p}_{i}(t)= & -p_{i}(t)+q_{i}(t), \\
\dot{q}_{i}(t)= & \left(-1+d_{i}-c_{i}\right) p_{i}(t)+\left(1-d_{i}\right) q_{i}(t) \\
& +\sum_{j=1}^{n} \tilde{a}_{i j}\left(p_{i}(t)\right) f_{j}\left(p_{j}(t)\right) \\
& +\sum_{j=1}^{n} \tilde{b}_{i j}\left(p_{i}(t)\right) f_{j}\left(p_{j}(t-\tau(t))\right)+I_{i},
\end{aligned}\right.
$$

with initial conditions

$$
\left\{\begin{array}{l}
p_{i}(s)=\phi_{i}(s), \\
q_{i}(s)=\phi_{i}(s)+\psi_{i}(s),
\end{array}\right.
$$

where $-\tau \leq s \leq 0, i=1, \cdots, n$.

By letting $p(t)=\left(p_{1}(t), p_{2}(t), \cdots, p_{n}(t)\right)^{T}, q(t)=$ $\left(q_{1}(t), q_{2}(t), \cdots, q_{n}(t)\right)^{T}$, system (6) can be written as the following compact form

$$
\left\{\begin{aligned}
\dot{p}(t)= & -p(t)+q(t), \\
\dot{q}(t)= & \left(-I_{n}+D-C\right) p(t)+\left(I_{n}-D\right) q(t) \\
& +\tilde{A}(p(t)) f(p(t)) \\
& +\tilde{B}(p(t)) f(p(t-\tau(t)))+I,
\end{aligned}\right.
$$


where $D=\operatorname{diag}\left\{d_{1}, \cdots, d_{n}\right\}, C=\operatorname{diag}\left\{c_{1}, \cdots c_{n}\right\}$, $\tilde{A}(p(t))=\left(\tilde{a}_{i j}\left(p_{i}(t)\right)\right)_{n \times n}, \tilde{B}(p(t))=\left(\tilde{b}_{i j}\left(p_{i}(t)\right)\right)_{n \times n}$. Define the corresponding response system for (1)

$$
\begin{aligned}
\frac{d^{2} x_{i}(t)}{d t^{2}}= & -d_{i} \frac{d x_{i}(t)}{d t}-c_{i} x_{i}(t)+\sum_{j=1}^{n} a_{i j}\left(x_{i}\right) f_{j}\left(x_{j}(t)\right) \\
& +\sum_{j=1}^{n} b_{i j}\left(x_{i}(t)\right) f_{j}\left(x_{j}(t-\tau(t))\right)+I_{i}+u_{i}(t) \\
& i=1, \cdots, n
\end{aligned}
$$

Applying the following variable transformation

$$
y_{i}(t)=x_{i}(t), z_{i}(t)=\frac{d x_{i}(t)}{d t}+x_{i}(t),
$$

the response system can be written as

$$
\left\{\begin{aligned}
\dot{y}(t)= & -y(t)+z(t) \\
\dot{z}(t)= & \left(-I_{n}+D-C\right) y(t)+\left(I_{n}-D\right) z(t) \\
& +\tilde{A}(y(t)) f(y(t)) \\
& +\tilde{B}(y(t)) f(y(t-\tau(t)))+I+u(t)
\end{aligned}\right.
$$

where $y(t)=\left[y_{1}(t), \cdots, y_{n}(t)\right]^{T}, z(t)=\left[z_{1}(t), \cdots\right.$, $\left.z_{n}(t)\right]^{T}$ are state variable of the response system, $u(t) \in R^{n}$ is input control to be designed later. $\tilde{A}(y(t))=\left(\tilde{a}_{i j}\left(y_{i}(t)\right)\right)_{n \times n}, \tilde{B}(y(t))=\left(\tilde{b}_{i j}\left(y_{i}(t)\right)\right)_{n \times n}$. The parameters of the response system are given as

$$
\begin{aligned}
& a_{i j}\left(y_{i}(t)\right)=\left\{\begin{array}{l}
\hat{a}_{i j},\left|y_{i}(t)\right| \leq T_{i}, \\
\check{a}_{i j},\left|y_{i}(t)\right|>T_{i},
\end{array}\right. \\
& b_{i j}\left(y_{i}(t)\right)=\left\{\begin{array}{l}
\hat{b}_{i j},\left|y_{i}(t)\right| \leq T_{i}, \\
\check{b}_{i j},\left|y_{i}(t)\right|>T_{i} .
\end{array}\right.
\end{aligned}
$$

By letting the synchronization error $e_{1}(t)=y(t)-$ $p(t), e_{2}(t)=z(t)-q(t)$, one can derive the following error system

$$
\left\{\begin{aligned}
\dot{e}_{1}(t)= & -e_{1}(t)+e_{2}(t) \\
\dot{e}_{2}(t)= & \left(-I_{n}+D-C\right) e_{1}(t)+\left(I_{n}-D\right) e_{2}(t) \\
& +\tilde{A}(y(t)) f(y(t))+\tilde{B}(y(t)) f(y(t-\tau(t))) \\
& -\tilde{A}(p(t)) f(p(t))-\tilde{B}(p(t)) f(p(t-\tau(t))) \\
& +u(t) .
\end{aligned}\right.
$$

\section{Definition and Lemmas}

In this Section, some elementary notations and lemmas are introduced which play an important role in the proof of the main results.
Definition 1 ([27]) Suppose $E \subseteq R^{n}$, then $x \mapsto F(x)$ is called a set-valued map from $E \mapsto R^{n}$, if for each point $x \in E$, there exists a nonempty set $F(x) \subseteq R^{n}$. A set-valued map $F$ with nonempty values is said to be upper semicontinuous at $x_{0} \in E$, if for any open set $N$ containing $F\left(x_{0}\right)$, there exists a neighbourhood $M$ of $x_{0}$ such that $F(M) \subseteq N$. The map $F(x)$ is said to have a closed image if for each $x \in E, F(x)$ is closed.

Definition 2 ([27]) For the system $d x / d t=f(t, x)$, $x \in R^{n}$, with discontinuous right-hand sides, a setvalued map is defined as

$$
F(t, x)=\cap_{\delta>0} \cap_{\mu(N)=0} \overline{c o}[f(B(x, \delta) \backslash N)],
$$

where $\overline{c o}[E]$ is the closure of the convex hull of set $E, B(x, \delta)=\{y:\|y-x\| \leq \delta\}$, and $\mu(N)$ is the Lebesgue measure of set $N$. A solution in Filippov's sense of the Cauchy problem for this system with initial condition $x(0)=x_{0}$ is an absolutely continuous function $x(t), t \in[0, T]$, which satisfies the differential inclusion

$$
\frac{d x}{d t} \in F(t, x), \quad t \in[0, T] .
$$

Definition 3 ([22])Suppose $W=\left(\omega_{i j}\right)_{n \times n}$ is a real matrix, then the matrix measure of $W$ is designed as follows

$$
\mu_{p}(W)=\lim _{h \rightarrow 0} \frac{\left\|I_{n}+h W\right\|_{p}-1}{h},
$$

where $\|\cdot\|_{p}$ is the induced matrix norm, $I_{n}$ is an identity matrix, $p=1,2, \infty$.

For $x \in R^{n}$, the vector norm are defined as

$$
\|x\|_{1}=\sum_{i=1}^{n}\left|x_{i}\right|,\|x\|_{2}=\sqrt{\sum_{i=1}^{n} x_{i}^{2}},\|x\|_{\infty}=\max _{1 \leq i \leq n}\left|x_{i}\right| .
$$

For matrix $W \in R^{n \times n}$, the matrix norm are defined as

$$
\begin{aligned}
\|W\|_{1} & =\max _{j} \sum_{i=1}^{n}\left|\omega_{i j}\right|, \quad\|W\|_{2}=\sqrt{\lambda_{\max }\left(W^{T} W\right)}, \\
\|W\|_{\infty} & =\max _{i} \sum_{j=1}^{n}\left|\omega_{i j}\right| .
\end{aligned}
$$

For $p=1,2, \infty$, the matrix measure can be calculated as

$$
\begin{aligned}
& \mu_{1}(W)=\max _{j}\left\{\omega_{j j}+\sum_{i=1, i \neq j}^{n}\left|\omega_{i j}\right|\right\}, \\
& \mu_{2}(W)=\lambda_{\max }\left(\frac{W^{T}+W}{2}\right), \\
& \mu_{\infty}(W)=\max _{i}\left\{\omega_{i i}+\sum_{j=1, i \neq j}^{n}\left|\omega_{i j}\right|\right\} .
\end{aligned}
$$


Definition 4 The drive-response systems (8) and (10) are said to be globally exponential synchronized if there exists constants $A \geq 0, \beta>0$ such that the condition

$$
\|e(t)\|_{1} \leq A e^{-\beta t} \sup _{-\tau \leq s \leq 0}\|e(s)\|_{1}
$$

holds for all $t \geq 0$, where $e(t)=\left(e_{1}^{T}(t), e_{2}^{T}(t)\right)^{T}$.

Lemma 1 ([28]) For any real vectors $x, y \in R^{n}$, the following inequality holds

$$
2 x^{T} y \leq x^{T} x+y^{T} y .
$$

Lemma 2 (Generalized Hanalay Inequality [25])If the nonnegative function $u(t)$ satisfies

$D^{+} u(t) \leq \gamma(t)+\alpha(t) u(t)+\beta(t) \sup _{t-\tau \leq s \leq t} u(s)$, $t \in\left[t_{0},+\infty\right)$,

where the continuous functions $\gamma(t) \geq 0, \alpha(t) \leq 0$, and $\beta(t) \geq 0$, if there exists constant $\delta$ to make the following inequality holds

$$
\alpha(t)+\beta(t) \leq-\delta<0, t \in\left[t_{0},+\infty\right),
$$

then we have

$$
u(t) \leq \frac{\gamma^{*}}{\delta}+\sup _{t-\tau \leq s \leq t} u(s) e^{-\mu^{*}\left(t-t_{0}\right)},
$$

where $\gamma^{*}=\sup _{t \in\left[t_{0},+\infty\right)} \gamma(t), \mu^{*}=\inf _{t \geq t_{0}}\{\mu(t)$ : $\left.\mu(t)+\alpha(t)+\beta(t) e^{\mu(t) \tau(t)}=0\right\}$.

\section{Main Results}

\subsection{Exponential Synchronization}

Constructing the following state feedback controller

$$
\begin{aligned}
u(t)= & -K_{1} e_{2}(t)-K_{2} \operatorname{sgn}\left(e_{2}(t)\right) \\
& -K_{3} \operatorname{sgn}\left(e_{2}(t)\right)\left|e_{1}(t)\right| \\
& -K_{4} \operatorname{sgn}\left(e_{2}(t)\right)\left|e_{1}(t-\tau(t))\right|,
\end{aligned}
$$

where $K_{1}, K_{2}, K_{3}, K_{4}$ are diagonal positive definite matrix.

For convenience, the following notation is given, where matrix $\bar{A}, \bar{B}, \bar{A}, \grave{A}, \bar{B}, \grave{B} \in R^{n \times n}$.

$$
\begin{aligned}
& \bar{A}=\left(\max \left\{\left|\hat{a}_{i j}\right|,\left|\check{a}_{i j}\right|\right\}\right)_{i j}, \bar{B}=\left(\max \left\{\left|\hat{b}_{i j}\right|,\left|\breve{b}_{i j}\right|\right\}\right)_{i j}, \\
& \dot{A}=\left(\max \left\{\hat{a}_{i j}, \check{a}_{i j}\right\}\right)_{i j}, \grave{A}=\left(\min \left\{\hat{a}_{i j}, \check{a}_{i j}\right\}\right)_{i j}, \\
& \dot{B}=\left(\max \left\{\hat{b}_{i j}, \check{b}_{i j}\right\}\right)_{i j}, \grave{B}=\left(\min \left\{\hat{b}_{i j}, \check{b}_{i j}\right\}\right)_{i j} .
\end{aligned}
$$

Theorem 3 Under Assumption 2.1 and 2.2, the drive-response system (8) and (10) will reach global exponential synchronization with the convergence rate $\beta>0$ if the following conditions hold

$$
\left\{\begin{array}{l}
\left|-I_{n}+D-C\right|-I_{n}+\bar{A} L-K_{3}+\beta I_{n} \leq 0 \\
\left|I_{n}-D\right|+I_{n}-K_{1}+\beta I_{n} \leq 0 \\
\bar{B} L-K_{4} \leq 0 \\
(\grave{A}-\grave{A}) M+(\grave{B}-\grave{B}) M-K_{2} 1 \leq 0,
\end{array}\right.
$$

where matrix $\left|-I_{n}+D-C\right|$ is obtained by taking absolute value of each elements of matrix $\left(-I_{n}+\right.$ $D-C) .1$ denotes the vector $(1,1, \cdots, 1)^{T} \in R^{n}$.

Proof. Choose a Lyapunov functional as

$$
V(t)=\|e(t)\|_{1}=\sum_{i=1}^{n}\left|e_{1 i}(t)\right|+\sum_{i=1}^{n}\left|e_{2 i}(t)\right|,
$$

where $e(t)=\left(e_{1}^{T}(t), e_{2}^{T}(t)\right)^{T}$.

Taking the derivative of $V(t)$ with respect to $t$ along the trajectory of the error system (12) yields

$$
\begin{aligned}
\dot{V}(t)= & \operatorname{sgn}\left(e_{1}^{T}(t)\right) \dot{e}_{1}(t)+\operatorname{sgn}\left(e_{2}^{T}(t)\right) \dot{e}_{2}(t) \\
\leq & s g n\left(e_{1}^{T}(t)\right)\left[-e_{1}(t)+e_{2}(t)\right]+\operatorname{sgn}\left(e_{2}^{T}(t)\right) \\
& {\left[\left(-I_{n}+D-C\right) e_{1}(t)+\left(I_{n}-D\right) e_{2}(t)\right] } \\
& +\operatorname{sgn}\left(e_{2}^{T}(t)\right)[\tilde{A}(y(t)) f(y(t))-\tilde{A}(p(t)) f(p(t)) \\
& +\tilde{B}(y(t)) f(y(t-\tau(t)))-\tilde{B}(p(t)) f(p(t-\tau(t)))] \\
& +\operatorname{sgn}\left(e_{2}^{T}(t)\right)\left[-K_{1} e_{2}(t)-K_{2} \operatorname{sgn}\left(e_{2}(t)\right)\right. \\
& \left.-K_{3} \operatorname{sgn}\left(e_{2}(t)\right)\left|e_{1}(t)\right|-K_{4} \operatorname{sgn}\left(e_{2}(t)\right)\left|e_{1}(t-\tau(t))\right|\right] \\
\leq & 1^{T}\left(\left|-I_{n}+D-C\right|-I_{n}\right)\left|e_{1}(t)\right| \\
& +1^{T}\left(\left|I_{n}-D\right|+I_{n}\right)\left|e_{2}(t)\right| \\
& +\operatorname{sgn}\left(e_{2}^{T}(t)\right)[\tilde{A}(y(t)) f(y(t))-\tilde{A}(p(t)) f(p(t)) \\
& +\tilde{B}(y(t)) f(y(t-\tau(t)))-\tilde{B}(p(t)) f(p(t-\tau(t)))] \\
& +\operatorname{sgn}\left(e_{2}^{T}(t)\right)\left[-K_{1} e_{2}(t)-K_{2} \operatorname{sgn}\left(e_{2}(t)\right)\right. \\
& -K_{3} \operatorname{sgn}\left(e_{2}(t)\right)\left|e_{1}(t)\right| \\
& \left.-K_{4} \operatorname{sgn}\left(e_{2}(t)\right)\left|e_{1}(t-\tau(t))\right|\right] .
\end{aligned}
$$

According to Assumption 2.1, 2.2 and Lemma 1, one has

$$
\begin{aligned}
& \operatorname{sgn}\left(e_{2}^{T}(t)\right)[\tilde{A}(y(t)) f(y(t))-\tilde{A}(p(t)) f(p(t))] \\
= & \operatorname{sgn}\left(e_{2}^{T}(t)\right) \tilde{A}(y(t))[f(y(t))-f(p(t))] \\
& +\operatorname{sgn}\left(e_{2}^{T}(t)\right)[\tilde{A}(y(t))-\tilde{A}(p(t))] f(p(t)) \\
\leq & 1^{T} \bar{A} L\left|e_{1}(t)\right|+1^{T}(\dot{A}-\grave{A}) M,
\end{aligned}
$$




$$
\begin{aligned}
& \operatorname{sgn}\left(e_{2}^{T}(t)\right)[\tilde{B}(y(t)) f(y(t-\tau(t))) \\
& -\tilde{B}(p(t)) f(p(t-\tau(t)))] \\
\leq & 1^{T} \bar{B} L\left|e_{1}(t-\tau(t))\right|+1^{T}(\dot{B}-\grave{B}) M
\end{aligned}
$$

where $L=\operatorname{diag}\left\{l_{1}, l_{2} \ldots l_{n}\right\}, M=\left(M_{1}, M_{2} \ldots M_{n}\right)^{T}$, $\left|e_{2}(t)\right|=\left(\left|e_{21}(t)\right|,\left|e_{22}(t)\right| \ldots\left|e_{2 n}(t)\right|\right)^{T}$.

Thus, it follows that

$$
\begin{aligned}
& \dot{V}(t)+\beta V(t) \\
\leq & 1^{T}\left(\left|-I_{n}+D-C\right|-I_{n}+\bar{A} L-K_{3}+\beta\right)\left|e_{1}(t)\right| \\
& +1^{T}\left(\left|I_{n}-D\right|+I_{n}-K_{1}+\beta\right)\left|e_{2}(t)\right| \\
& +1^{T}\left(\bar{B} L-K_{4}\right)\left|e_{1}(t-\tau(t))\right| \\
& +1^{T}\left[(\bar{A}-\grave{A}) M+(\bar{B}-\grave{B}) M-K_{2} 1\right] .
\end{aligned}
$$

For the purpose of $\dot{V}(t) \leq 0$, we can let

$$
\left\{\begin{array}{l}
\left|-I_{n}+D-C\right|-I_{n}+\bar{A} L-K_{3}+\beta I_{n} \leq 0 \\
\left|I_{n}-D\right|+I_{n}-K_{1}+\beta I_{n} \leq 0 \\
\bar{B} L-K_{4} \leq 0 \\
(\grave{A}-\grave{A}) M+(\grave{B}-\grave{B}) M-K_{2} 1 \leq 0
\end{array}\right.
$$

It follows that

$$
\dot{V}(t)+\beta V(t) \leq 0,
$$

thus, we have $\frac{1}{2}\|e(t)\|_{1}=V(t) \leq V(0) e^{-\beta t} \leq$ $\frac{1}{2} e^{-\beta t} \sup _{-\tau \leq s \leq 0}\|e(s)\|_{1}$. According to Definition 4, the drive-response system (8) and (10) will achieve global exponential synchronization under controller (13).

Remark 4.1 In conventional state feedback control, the control goal can be achieved. However, the obtained control gains may be much larger than actual needs, which will increase the cost of control. To overcome this drawback, the adaptive control method is investigated.

For convenience of discussion, we consider the following error system.

$$
\left\{\begin{aligned}
\dot{e}_{1 i}(t)= & -e_{1 i}(t)+e_{2 i}(t) \\
\dot{e}_{2 i}(t)= & \left(-1+d_{i}-c_{i}\right) e_{1 i}(t)+\left(1-d_{i}\right) e_{2 i}(t) \\
& +\sum_{i=1}^{n} \tilde{a}_{i j}\left(y_{i}(t)\right) f_{j}\left(y_{j}(t)\right) \\
& -\sum_{i=1}^{n} \tilde{a}_{i j}\left(p_{i}(t)\right) f_{j}\left(p_{j}(t)\right) \\
& +\sum_{i=1}^{n} \tilde{b}_{i j}\left(y_{i}(t)\right) f_{j}\left(y_{j}(t-\tau(t))\right) \\
& -\sum_{i=1}^{n} \tilde{b}\left(p_{i}(t)\right) f_{j}\left(p_{j}(t-\tau(t))\right)+u_{i}(t)
\end{aligned}\right.
$$

Theorem 4 Under Assumption 2.1 and 2.2, the drive-response system (8) and (10) will reach global exponential synchronization with the convergence rate $\beta>0$ under the adaptive controller

$$
\left\{\begin{array}{l}
u_{i}(t)=-k_{1 i}(t) e_{2 i}(t)-k_{2 i}(t) \operatorname{sgn}\left(e_{2 i}(t)\right) \\
-k_{3 i}(t) \operatorname{sgn}\left(e_{2 i}(t)\right)\left|e_{1 i}(t)\right| \\
-k_{4 i}(t) \operatorname{sgn}\left(e_{2 i}(t)\right)\left|e_{1 i}(t-\tau(t))\right| \\
\dot{k}_{1 i}(t)=\varepsilon_{i} e^{\beta t}\left|e_{2 i}(t)\right| \\
\dot{k}_{2 i}(t)=\xi_{i} e^{\beta t} \\
\dot{k}_{3 i}(t)=\gamma_{i} e^{\beta t}\left|e_{1 i}(t)\right| \\
\dot{k}_{4 i}(t)=\eta_{i} e^{\beta t}\left|e_{1 i}(t-\tau(t))\right|
\end{array}\right.
$$

where $i=1,2, \cdots, n, \varepsilon_{i}, \xi_{i}, \gamma_{i}, \eta_{i}$ are positive constants, the initial value of $k_{1 i}(t), k_{2 i}(t), k_{3 i}(t), k_{4 i}(t)$ are some small positive constants.

Proof. Choose the Lyapunov functional as

$$
V(t)=V_{1}(t)+V_{2}(t)
$$

where

$$
V_{1}(t)=e^{\beta t} \sum_{i=1}^{n}\left(\left|e_{1 i}(t)\right|+\left|e_{2 i}(t)\right|\right),
$$

$$
\begin{aligned}
V_{2}(t)= & \Sigma_{i=1}^{n} \frac{1}{2 \varepsilon_{i}}\left(k_{1 i}(t)-k_{1 i}\right)^{2}+\sum_{i=1}^{n} \frac{1}{2 \xi_{i}}\left(k_{2 i}(t)-k_{2 i}\right)^{2} \\
& +\sum_{i=1}^{n} \frac{1}{2 \gamma_{i}}\left(k_{3 i}(t)-k_{3 i}\right)^{2}+\sum_{i=1}^{n} \frac{1}{2 \eta_{i}}\left(k_{4 i}(t)-k_{4 i}\right)^{2}
\end{aligned}
$$

where $k_{1 i}, k_{2 i}, k_{3 i}, k_{4 i}$ are positive constants to be determined later.

Taking the derivative of $V(t)$ with respect to $t$ along the trajectory of the error system (18) yields 


$$
V(0)=\|e(0)\|_{1}+\leq \sup _{-\tau \leq s \leq 0}\|e(s)\|_{1} .
$$

$$
\begin{aligned}
& \dot{V}_{1}(t)=\beta e^{\beta t} \Sigma_{i=1}^{n}\left(\left|e_{1 i}(t)\right|+\left|e_{2 i}(t)\right|\right) \\
& +e^{\beta t} \sum_{i=1}^{n}\left(\operatorname{sgn}\left(e_{1 i}(t)\right) \dot{e}_{1 i}(t)+\operatorname{sgn}\left(e_{2 i}(t)\right) \dot{e}_{2 i}(t)\right) \\
& \leq \beta e^{\beta t} \sum_{i=1}^{n}\left(\left|e_{1 i}(t)\right|+\left|e_{2 i}(t)\right|\right) \\
& +e^{\beta t} \sum_{i=1}^{n} \operatorname{sgn}\left(e_{1 i}(t)\left(-e_{1 i}(t)+e_{2 i}(t)\right)\right. \\
& +e^{\beta t} \sum_{i=1}^{n} \operatorname{sgn}\left(e _ { 2 i } ( t ) \left[\left(-1+d_{i}-c_{i}\right) e_{1 i}(t)\right.\right. \\
& \left.+\left(1-d_{i}\right) e_{2 i}(t)\right]+e^{\beta t} \Sigma_{i=1}^{n} \operatorname{sgn}\left(e_{2 i}(t)\right) . \\
& V(0)=\|e(0)\|_{1}+\sum_{i=1}^{n} \frac{1}{2 \varepsilon_{i}}\left(k_{1 i}(0)-k_{1 i}\right)^{2} \\
& +\sum_{i=1}^{n} \frac{1}{2 \xi_{i}}\left(k_{2 i}(0)-k_{2 i}\right)^{2}+\sum_{i=1}^{n} \frac{1}{2 \gamma_{i}}\left(k_{3 i}(0)\right. \\
& \left.-k_{3 i}\right)^{2}+\Sigma_{i=1}^{n} \frac{1}{2 \eta_{i}}\left(k_{4 i}(0)-k_{4 i}\right)^{2}
\end{aligned}
$$$$
\sum_{j=1}^{n}\left[\tilde{a}_{i j}\left(y_{i}\right) f_{j}\left(y_{j}(t)\right)-\tilde{a}_{i j}\left(p_{i}\right) f_{j}\left(p_{j}(t)\right)\right.
$$$$
+e^{\beta t} \sum_{i=1}^{n} \operatorname{sgn}\left(e_{2 i}(t)\right)\left[-k_{1 i}(t) e_{2 i}(t)\right.
$$$$
-k_{2 i}(t) \operatorname{sgn}\left(e_{2 i}(t)\right)-k_{3 i}(t) \operatorname{sgn}\left(e_{2 i}(t)\right)\left|e_{1 i}(t)\right|
$$$$
\left.-k_{4 i}(t) \operatorname{sgn}\left(e_{2 i}(t)\right)\left|e_{1 i}(t-\tau(t))\right|\right]
$$$$
=e^{\beta t} \Sigma_{i=1}^{n}\left[\beta-1+\left|-1+d_{i}-c_{i}\right|\right.
$$$$
\left.+\sum_{j=1}^{n} \bar{a}_{j i} l_{i}-k_{3 i}(t)\right]\left|e_{1 i}(t)\right|
$$$$
+e^{\beta t} \sum_{i=1}^{n}\left[\beta+1+\left|1-d_{i}\right|-k_{1 i}(t)\right]\left|e_{2 i}(t)\right|
$$$$
+e^{\beta t} \sum_{i=1}^{n}\left[-k_{4 i}(t)+\sum_{j=1}^{n} \bar{b}_{j i} l_{i}\right]\left|e_{1 i}(t-\tau(t))\right|
$$$$
+e^{\beta t} \sum_{i=1}^{n}\left[-k_{2 i}(t)+\sum_{j=1}^{n}\left(\hat{a}_{i j}-\breve{a}_{i j}\right.\right.
$$$$
\left.\left.+\hat{b}_{i j}-\breve{b}_{i j}\right) M_{j}\right]
$$

If $\sup _{-\tau \leq s \leq 0}\|e(s)\|_{1} \neq 0$, then there exists positive constants $\rho$ such that

$$
\begin{aligned}
& \left.+\tilde{b}_{i j}\left(y_{i}\right) f_{j}\left(y_{j}(t-\tau(t))\right)-\tilde{b}_{i j}\left(p_{i}\right) f_{j}\left(p_{j}(t-\tau(t))\right)\right] \\
& \sum_{i=1}^{n} \frac{1}{2 \varepsilon_{i}}\left(k_{1 i}(0)-k_{1 i}\right)^{2}+\sum_{i=1}^{n} \frac{1}{2 \xi_{i}}\left(k_{2 i}(0)-k_{2 i}\right)^{2} \\
& +\sum_{i=1}^{n} \frac{1}{2 \gamma_{i}}\left(k_{3 i}(0)-k_{3 i}\right)^{2}+\sum_{i=1}^{n} \frac{1}{2 \eta_{i}}\left(k_{4 i}(0)-k_{4 i}\right)^{2} \\
& \leq \rho \sup _{-\tau \leq s \leq 0}\|e(s)\|_{1}
\end{aligned}
$$

Therefore, $\|e(t)\|_{1} \leq e^{-\beta t}(1+\rho) \sup _{-\tau \leq s \leq 0}\|e(s)\|_{1}$. According to Definition 4, the drive-response system (8) and (10) will achieve global exponential synchronization under adaptive controller (19).

Remark 4.2 It is well known that the sign function in controller (19) will introduce chattering effects to

$$
\begin{aligned}
\dot{V}_{2}(t)= & e^{\beta t} \sum_{i=1}^{n}\left(k_{1 i}(t)-k_{1 i}\right)\left|e_{2 i}(t)\right|+e^{\beta t} \sum_{i=1}^{n}\left(k_{2 i}(t)\right. \\
& \left.-k_{2 i}\right)+e^{\beta t} \sum_{i=1}^{n}\left(k_{3 i}(t)-k_{3 i}\right)\left|e_{1 i}(t)\right| \\
& +e^{\beta t} \sum_{i=1}^{n}\left(k_{4 i}(t)-k_{4 i}\right)\left|e_{1 i}(t-\tau(t))\right|
\end{aligned}
$$

Therefore,

$$
\begin{aligned}
\dot{V}(t) \leq & e^{\beta t} \sum_{i=1}^{n}\left[\beta-1+\left|-1+d_{i}-c_{i}\right|+\sum_{j=1}^{n} \bar{a}_{j i} l_{i}\right. \\
& \left.-k_{3 i}\right]\left|e_{1 i}(t)\right|+e^{\beta t} \sum_{i=1}^{n}\left[\beta+1+\left|1-d_{i}\right|\right. \\
& \left.-k_{1 i}\right]\left|e_{2 i}(t)\right|+e^{\beta t} \sum_{i=1}^{n}\left[-k_{4 i}+\sum_{j=1}^{n} \bar{b}_{j i} l_{i}\right] \\
& \cdot\left|e_{1 i}(t-\tau(t))\right|+e^{\beta t} \sum_{i=1}^{n}\left[-k_{2 i}\right. \\
& \left.+\sum_{j=1}^{n}\left(\hat{a}_{i j}-\breve{a}_{i j}+\hat{b}_{i j}-\breve{b}_{i j}\right) M_{j}\right]
\end{aligned}
$$

Now, choose parameters as

$$
\left\{\begin{array}{l}
k_{1 i}=\beta+1+\left|1-d_{i}\right|, \\
k_{2 i}=\sum_{j=1}^{n}\left(\hat{a}_{i j}-\breve{a}_{i j}+\hat{b}_{i j}-\breve{b}_{i j}\right) M_{j}, \\
k_{3 i}=\beta-1+\left|-1+d_{i}-c_{i}\right|+\Sigma_{j=1}^{n} \bar{a}_{j i} l_{i}, \\
k_{4 i}=\sum_{j=1}^{n} \bar{b}_{j i} l_{i} .
\end{array}\right.
$$

It follows that $\dot{V}(t) \leq 0$. Thus, $e^{\beta t}\|e(t)\|_{1}=V(t) \leq$ system state. To weaken the chattering effects, the following saturation functions can be employed to replace the sign function $\operatorname{sgn}\left(e_{2 i}(t)\right)$.

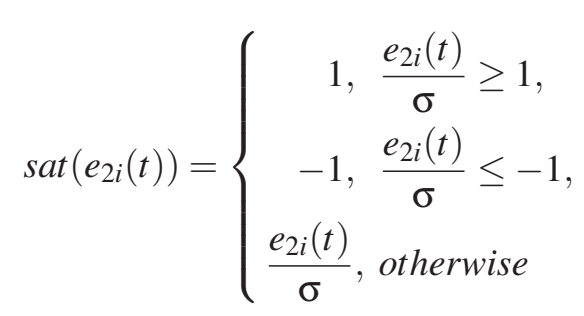

where $i=1,2, \cdots, n$, and $\sigma$ is a small positive constant.

\subsection{Quasi-Synchronization}

For the system with unmatched parameters, synchronization will not tend to zero as time goes to infinity, so completely synchronization can not be achieved. However, by designing appropriate control input, the synchronization error can be made into an arbitrarily small range as time tend to infinity to satisfy our needs. This phenomenon is called the quasi-synchronization. Consider the following 
inertial memristive neural network

$$
\begin{aligned}
\frac{d^{2} s_{i}(t)}{d t^{2}}= & -d_{i} \frac{d s_{i}(t)}{d t}-c_{i} s_{i}(t)+\sum_{j=1}^{n} a_{i j}\left(s_{i}(t)\right) f_{j}\left(s_{j}(t)\right) \\
& +\sum_{j=1}^{n} b_{i j}\left(s_{i}(t)\right) f_{j}\left(s_{j}(t-\tau(t))\right)+I_{i},
\end{aligned}
$$

where

$$
\begin{aligned}
& a_{i j}\left(s_{i}(t)\right)=\left\{\begin{array}{l}
\hat{a}_{i j},\left|s_{i}(t)\right| \leq T_{i}, \\
\check{a}_{i j},\left|s_{i}(t)\right|>T_{i},
\end{array}\right. \\
& b_{i j}\left(s_{i}(t)\right)=\left\{\begin{array}{l}
\hat{b}_{i j},\left|s_{i}(t)\right| \leq T_{i}, \\
\breve{b}_{i j},\left|s_{i}(t)\right|>T_{i} .
\end{array}\right.
\end{aligned}
$$

Due to the parameter mismatch, the response system is given as

$$
\begin{aligned}
\frac{d^{2} x_{i}(t)}{d t^{2}}= & -d_{i} \frac{d x_{i}(t)}{d t}-c_{i} x_{i}(t)+\sum_{j=1}^{n} a_{i j}^{*}\left(x_{i}(t)\right) f_{j}\left(x_{j}(t)\right) \\
& +\sum_{j=1}^{n} b_{i j}^{*}\left(x_{i}(t)\right) f_{j}\left(x_{j}(t-\tau(t))\right)+I_{i}+u_{i}(t)
\end{aligned}
$$

where

$$
\begin{aligned}
& a_{i j}^{*}\left(x_{i}(t)\right)=\left\{\begin{array}{l}
\hat{a}_{i j}^{*},\left|x_{i}(t)\right| \leq T_{i}, \\
\check{a}_{i j}^{*},\left|x_{i}(t)\right|>T_{i},
\end{array}\right. \\
& b_{i j}^{*}\left(x_{i}(t)\right)=\left\{\begin{array}{l}
\hat{b}_{i j}^{*},\left|x_{i}(t)\right| \leq T_{i}, \\
\check{b}_{i j}^{*},\left|x_{i}(t)\right|>T_{i}
\end{array}\right.
\end{aligned}
$$

According to the previous discussion, the driveresponse system (26) and (28) can be transformed into the following second-order system

$$
\begin{aligned}
& \left\{\begin{aligned}
\dot{p}(t)= & -p(t)+q(t), \\
\dot{q}(t)= & \left(-I_{n}+D-C\right) p(t)+\left(I_{n}-D\right) q(t) \\
& +\tilde{A} f(p(t))+\tilde{B} f(p(t-\tau(t)))+I .
\end{aligned}\right. \\
& \left\{\begin{aligned}
\dot{y}(t)= & -y(t)+z(t), \\
\dot{z}(t)= & \left(-I_{n}+D-C\right) y(t)+\left(I_{n}-D\right) z(t) \\
& +\tilde{A}^{*} f(y(t))+\tilde{B}^{*} f(y(t-\tau(t))) \\
& +I+u(t),
\end{aligned}\right.
\end{aligned}
$$

where $\tilde{A}=\left(\tilde{a}_{i j}\left(p_{i}(t)\right)\right)_{n \times n}, \quad \tilde{B}=\left(\tilde{b}_{i j}\left(p_{i}(t)\right)\right)_{n \times n}$, $\tilde{A}^{*}=\left(\tilde{a}_{i j}^{*}\left(y_{i}(t)\right)\right)_{n \times n}, \tilde{B}^{*}=\left(\tilde{b}_{i j}^{*}\left(y_{i}(t)\right)\right)_{n \times n}$.

By letting $e_{1}(t)=y(t)-p(t), e_{2}(t)=z(t)-q(t)$, the following error system is achieved

$$
\left\{\begin{aligned}
\dot{e}_{1}(t)= & -e_{1}(t)+e_{2}(t), \\
\dot{e}_{2}(t)= & \left(-I_{n}+D-C\right) e_{1}(t)+\left(I_{n}-D\right) e_{2}(t) \\
& +\tilde{A}^{*} f(y(t))+\tilde{B}^{*} f(y(t-\tau(t))) \\
& -\tilde{A} f(p(t))-\tilde{B} f(p(t-\tau(t)))+u(t) .
\end{aligned}\right.
$$

Define $e(t)=\left(e_{1}(t), e_{2}(t)\right)^{T}$, the error system (32) can be written as

$$
\dot{e}(t)=G e(t)+\left(\begin{array}{c}
0 \\
\tilde{A}^{*} f(y(t))-\tilde{A} f(p(t)) \\
+\tilde{B}^{*} f(y(t-\tau(t))) \\
-\tilde{B} f(p(t-\tau(t)))+u(t)
\end{array}\right) .
$$

By designing the following state feedback controller

$$
u(t)=-K_{2} e_{2}(t),
$$

where $K_{2}=\operatorname{diag}\left(k_{1} \cdots k_{n}\right) \in R^{n \times n}$, we get the error system as follows

$\dot{e}(t)=G e(t)+\left(\begin{array}{c}0 \\ \tilde{A}^{*} f(y(t))-\tilde{A} f(p(t)) \\ +\tilde{B}^{*} f(y(t-\tau(t))) \\ -\tilde{B} f(p(t-\tau(t)))\end{array}\right)-K e(t)$,

where $\quad G=\left(\begin{array}{cc}-I_{n} & I_{n} \\ -I_{n}+D-C & I_{n}-D\end{array}\right), \quad K=$ $\operatorname{diag}\left(0 \cdots 0, k_{1} \cdots k_{n}\right) \in R^{2 n \times 2 n}$.

According to the switching character of memristor, Each of $\tilde{A}, \tilde{A}^{*}, \tilde{B}, \tilde{B}^{*}$ has $2^{n}$ possible values. For the convenience of following discussion, we make some notations as follows $\left\|\bar{A}^{*}\right\|_{p}=\max \left\{\left\|\tilde{A}^{*}\right\|_{p}\right\},\left\|\bar{B}^{*}\right\|_{p}=\max \left\{\left\|\tilde{B}^{*}\right\|_{p}\right\}$, $\|\Delta A\|_{p}=\max \left\{\left\|\tilde{A}^{*}-\tilde{A}\right\|_{p}\right\},\|\Delta B\|_{p}=\max \left\{\| \tilde{B}^{*}-\right.$ $\left.\tilde{B} \|_{p}\right\}, p=1,2, \infty$.

The following assumptions can be directly achieved from Assumption 2.1 and Assumption 2.2

Assumption 4.1 There exists positive constant $l^{p}$ $(p=1,2, \infty)$ such that

$$
\left\|f\left(s_{1}\right)-f\left(s_{2}\right)\right\|_{p} \leq l^{p}\left\|s_{1}-s_{2}\right\|_{p}, \forall s_{1}, s_{2} \in R^{n} .
$$

Assumption 4.2 There exists positive constants $M_{p}>0$ such that $\|f(\cdot)\|_{p} \leq M_{p}, p=1,2, \infty$.

Theorem 5 Under Assumption 4.1 and 4.2, if the following inequality holds

$$
\mu_{p}(G-K)+l^{p}\left\|\bar{A}^{*}\right\|_{p}+l^{p}\left\|\bar{B}^{*}\right\|_{p} \leq-\delta<0,
$$


Then, the synchronization error converges to the following set

$$
M=\left\{e(t) \in R^{n} \mid\|e(t)\|_{p} \leq \gamma^{*} / \delta\right\},
$$

where $\gamma^{*}=\sup _{t \geq 0}\left\{M_{p}\left(\|\Delta A\|_{p}+\|\Delta B\|_{p}\right)\right\}<\infty, p=$ $1,2, \infty$. It means that drive system (30) and response system (31) achieve quasi-synchronization with an error level $\gamma^{*} / \delta$.

Proof. Consider the following Lyapunov functional

$$
V(t)=\|e(t)\|_{p}, p=1,2, \infty,
$$

Taking the derivative of $V(t)$ with respect to $t$ along the trajectory of the error system (32) yields

$$
\begin{aligned}
D^{+} V(t)= & \lim _{h \rightarrow 0^{+}} \frac{\|e(t+h)\|_{p}-\|e(t)\|_{p}}{h} \\
= & \lim _{h \rightarrow 0^{+}} \frac{\|e(t)+h \dot{e}(t)+o(h)\|_{p}-\|e(t)\|_{p}}{h} \\
= & \lim _{h \rightarrow 0^{+}} \frac{1}{h}\{\| e(t) \\
& +h\left(\begin{array}{c}
\tilde{A}^{*} f(y(t))-\tilde{A} f(p(t)) \\
+\tilde{B}^{*} f(y(t-\tau(t))) \\
-\tilde{B} f(p(t-\tau(t)))
\end{array}\right) \\
& \left.+h G e(t)-h K e(t)+o(h)\left\|_{p}-\right\| e(t) \|_{p}\right\} \\
\leq & l i m_{h \rightarrow 0^{+}} \frac{1}{h}\left\{\left\|I_{2 n}+h(G-K)\right\|_{p}-1\right\}\|e(t)\|_{p} \\
& +\left\|\tilde{A}^{*} f(y(t))-\tilde{A} f(p(t))\right\|_{p} \\
& +\| \tilde{B}^{*} f(y(t-\tau(t)))-\tilde{B} f\left(p(t-\tau(t)) \|_{p}\right. \\
\leq & \mu_{p}(G-K)\|e(t)\|_{p}+l^{p}\left\|\tilde{A}^{*}\right\|_{p}\left\|_{1}(t)\right\|_{p} \\
& +\left\|\left(\tilde{A}^{*}-\tilde{A}\right)\right\|_{p}\|f(p(t))\|_{p} \\
& +l^{p}\left\|\tilde{B}^{*}\right\|_{p}\left\|e_{1}(t-\tau(t))\right\|_{p} \\
& +\left\|\left(\tilde{B}^{*}-\tilde{B}\right)\right\|_{p}\|f(p(t-\tau(t)))\|_{p} \\
\leq & \mu_{p}(G-K)\|e(t)\|_{p}+l^{p}\left\|\tilde{A}^{*}\right\|_{p}\|e(t)\|_{p} \\
& +l^{p}\left\|\tilde{B}^{*}\right\|_{p}\|e(t-\tau(t))\|_{p} \\
& +\left\|\left(\tilde{A}^{*}-\tilde{A}\right)\right\|_{p}\|f(p(t))\|_{p} \\
& +\left\|\left(\tilde{B}^{*}-\tilde{B}\right)\right\|_{p}\|f(p(t-\tau(t)))\|_{p} \\
\leq & \left(\mu_{p}(G-K)+l^{p}\left\|\tilde{A}^{*}\right\|_{p}\right)\|e(t)\|_{p} \\
& +l^{p}\left\|\tilde{B}^{*}\right\|_{p} \quad \sup _{t-\tau \leq s \leq t}\|e(s)\|_{p} \\
& +\|\Delta A\|_{p} M_{p}+\|\Delta B\|_{p} M_{p} . \\
&
\end{aligned}
$$

Let $\alpha(t)=\mu_{p}(G-K)+l^{p}\left\|\bar{A}^{*}\right\|_{p}, \beta(t)=l^{p}\left\|\bar{B}^{*}\right\|_{p}$, $\gamma(t)=\|\Delta A\|_{p} M_{p}+\|\Delta B\|_{p} M_{p}$, we can get that

$$
D^{+} V(t) \leq \gamma(t)+\alpha(t) V(t)+\beta(t) \sup _{t-\tau \leq s \leq t} V(s) .
$$

According to the condition of Theorem 5, we have $\alpha(t)+\beta(t) \leq-\delta<0$. Using Lemma 2 , it follows that

$$
\|e(t)\|_{p} \leq \frac{\gamma^{*}}{\delta}+\sup _{-\tau \leq s \leq 0}\|e(s)\|_{p} e^{-\mu^{*} t},
$$

where $\mu^{*}=\inf f_{t \geq 0}\left\{\mu(t): \mu(t)+\alpha(t)+\beta(t) e^{\mu(t) \tau}=\right.$ $0\}>0$. When $t \rightarrow \infty, \sup _{-\tau \leq s \leq 0}\|e(s)\|_{p} e^{-\mu^{*} t} \rightarrow 0$. Therefore, the system error converges to the set $M=\left\{e(t) \in R^{n} \mid\|e(t)\|_{p} \leq \gamma^{*} / \delta\right\}$, which can be arbitrarily small by letting $\delta$ be sufficiently large. According to above discussion, systems (30) and (31) reach quasi-synchronization with an error level $\gamma^{*} / \delta$.

Remark 4.3 In Theorem (5), the synchronization error $\gamma^{*} / \delta$ will not tend to 0 as $t \rightarrow \infty$. However, it can be made into an arbitrarily small range by letting elements in control gain matrix $K$ sufficiently large. Though weaker than complete synchronization, quasi-synchronization has its advantage and it can be widely applied in practical applications due to its lower costs. Besides, in many real situations, we only need the synchronization error be controlled in an acceptable region instead of tending to 0.

Remark 4.4 The matrix measure approach has the following advantages: firstly, we do not need to construct Lyapunov functional. Secondly, the effects of positive values and negative values of the matrix can be trade off by matrix measure method. It is more powerful when dealing with the case when the connection weights contain both positive and negative values, which corresponds to the excitation and inhibition in neurons, respectively.

\section{Numerical examples}

In order to show the effectiveness of the theoretical results, the following numerical examples are given. Firstly, we consider the globally exponential synchronization.

Example 1 Consider the drive inertial memristive neural network 


$$
\left\{\begin{aligned}
\dot{p}_{1}(t)= & -p_{1}(t)+q_{1}(t), \\
\dot{p}_{2}(t)= & -p_{2}(t)+q_{2}(t), \\
\dot{q}_{1}(t)= & \left(-1+d_{1}-c_{1}\right) p_{1}(t)+\left(1-d_{1}\right) q_{1}(t) \\
& +a_{11}\left(p_{1}(t)\right) f_{1}\left(p_{1}(t)\right) \\
& +a_{12}\left(p_{1}(t)\right) f_{2}\left(p_{2}(t)\right) \\
& +b_{11}\left(p_{1}(t)\right) f_{1}\left(p_{1}(t-\tau(t))\right) \\
& +b_{12}\left(p_{1}(t)\right) f_{2}\left(p_{2}(t-\tau(t))\right)+I_{1}, \\
\dot{q}_{2}(t)= & \left.-1+d_{2}-c_{2}\right) p_{2}(t)+\left(1-d_{2}\right) q_{2}(t) \\
& +a_{21}\left(p_{2}(t)\right) f_{1}\left(p_{1}(t)\right) \\
& +a_{22}\left(p_{2}(t)\right) f_{2}\left(p_{2}(t)\right) \\
& +b_{21}\left(p_{2}(t)\right) f_{1}\left(p_{1}(t-\tau(t))\right) \\
& +b_{22}\left(p_{2}(t)\right) f_{2}\left(p_{2}(t-\tau(t))\right)+I_{2},
\end{aligned}\right.
$$

the memristive connection weight are as follows

$$
\begin{aligned}
& a_{11}\left(p_{1}\right)=\left\{\begin{array}{c}
1,\left|p_{1}\right| \leq 1, \\
-1,\left|p_{1}\right|>1,
\end{array} \quad a_{12}\left(p_{1}\right)=\left\{\begin{array}{l}
2,\left|p_{1}\right| \leq 1, \\
1,\left|p_{1}\right|>1,
\end{array}\right.\right. \\
& a_{21}\left(p_{2}\right)=\left\{\begin{array}{c}
1,\left|p_{2}\right| \leq 1, \\
-1,\left|p_{2}\right|>1,
\end{array} \quad a_{22}\left(p_{2}\right)=\left\{\begin{array}{l}
2,\left|p_{2}\right| \leq 1, \\
1,\left|p_{2}\right|>1,
\end{array}\right.\right. \\
& b_{11}\left(p_{1}\right)=\left\{\begin{array}{c}
1,\left|p_{1}\right| \leq 1, \\
-2,\left|p_{1}\right|>1,
\end{array} \quad b_{12}\left(p_{1}\right)=\left\{\begin{array}{l}
1,\left|p_{1}\right| \leq 1, \\
2,\left|p_{1}\right|>1,
\end{array}\right.\right. \\
& b_{21}\left(p_{2}\right)=\left\{\begin{array}{l}
2,\left|p_{2}\right| \leq 1, \\
1,\left|p_{2}\right|>1,
\end{array} b_{22}\left(p_{2}\right)=\left\{\begin{array}{c}
2,\left|p_{2}\right| \leq 1, \\
-1,\left|p_{2}\right|>1,
\end{array}\right.\right.
\end{aligned}
$$

the response system is

$$
\left\{\begin{aligned}
\dot{y}_{1}(t)= & -y_{1}(t)+z_{1}(t), \\
\dot{y}_{2}(t)= & -y_{2}(t)+z_{2}(t), \\
\dot{z}_{1}(t)= & \left(-1+d_{1}-c_{1}\right) y_{1}(t)+\left(1-d_{1}\right) z_{1}(t) \\
& +a_{11}\left(y_{1}(t)\right) f_{1}\left(y_{1}(t)\right)+a_{12}\left(y_{1}(t)\right) f_{2}\left(y_{2}(t)\right) \\
& +b_{11}\left(y_{1}(t)\right) f_{1}\left(y_{1}(t-\tau(t))\right) \\
& +b_{12}\left(y_{1}(t)\right) f_{2}\left(y_{2}(t-\tau(t))\right) \\
& +I_{1}-k_{11} e_{21}(t)-k_{21} \operatorname{sgn}\left(e_{21}(t)\right) \\
& -k_{31} \operatorname{sgn}\left(e_{21}(t)\right)\left|e_{11}(t)\right| \\
& -k_{41} \operatorname{sgn}\left(e_{21}(t)\right)\left|e_{11}(t-\tau(t))\right|, \\
\dot{z}_{2}(t)= & \left.-1+d_{2}-c_{2}\right) y_{2}(t)+\left(1-d_{2}\right) z_{2}(t) \\
& +a_{21}\left(y_{2}(t)\right) f_{1}\left(y_{1}(t)\right)+a_{22}\left(y_{2}(t)\right) f_{2}\left(y_{2}(t)\right) \\
& +b_{21}\left(y_{2}(t)\right) f_{1}\left(y_{1}(t-\tau(t))\right) \\
& +b_{22}\left(y_{2}(t)\right) f_{2}\left(y_{2}(t-\tau(t))\right) \\
& +I_{2}-k_{12} e_{22}(t)-k_{22} \operatorname{sgn}\left(e_{22}(t)\right) \\
& -k_{32} \operatorname{sgn}\left(e_{22}(t)\right)\left|e_{12}(t)\right| \\
& -k_{42} \operatorname{sgn}\left(e_{22}(t)\right)\left|e_{12}(t-\tau(t))\right|,
\end{aligned}\right.
$$

Choose the activation functions $f_{1}(x)=f_{2}(x)=$ $\sin x, \beta=1, \tau(t)=0.8+0.2 \sin 3 t$, external inputs are $I_{1}=I_{2}=0$. Let $c_{1}=2, c_{2}=1, d_{1}=d_{2}=1$. According to Assumption 2.1 and 2.2, $l_{1}=l_{2}=$ $1, M_{1}=M_{2}=1$. By simple computation, it can be obtained that $k_{11} \geq 2, k_{12} \geq 2, k_{21} \geq 10, k_{22} \geq$ $10, k_{31} \geq 5, k_{32} \geq 4, k_{41} \geq 4, k_{42} \geq 4$.

When control gains are chosen as $k_{11}=4, k_{12}=$ $4, k_{21}=12, k_{22}=12, k_{31}=6, k_{32}=6, k_{41}=6, k_{42}=$ 6 , the condition of Theorem 4 holds. Choose the initial condition as $p_{1}(t)=3, p_{2}(t)=2, q_{1}(t)=$ $4, q_{2}(t)=0, \quad y_{1}(t)=-2, y_{2}(t)=-1, z_{1}(t)=$ $-1, z_{2}(t)=2, t \in[-1,0]$. The results of the simulation are shown in Figure 1, synchronization error $e_{11}, e_{12}, e_{21}, e_{22}$ will converge to 0 as $t \rightarrow \infty$, which verify the effectiveness of Theorem 4.

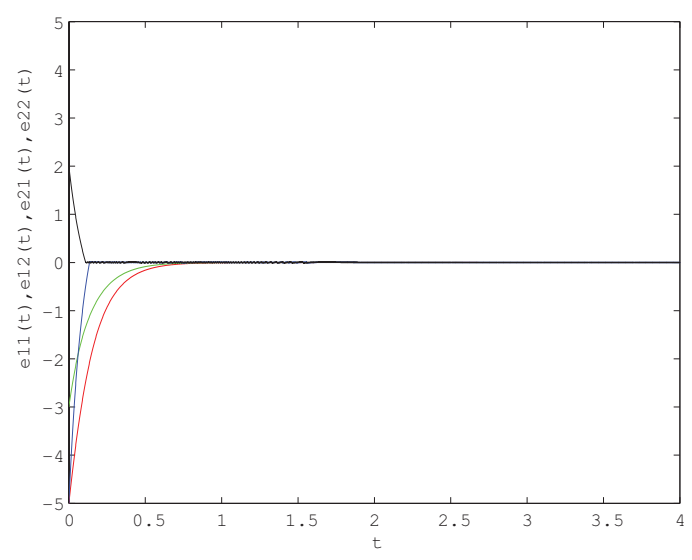

Figure 1. Synchronization error $e_{11}, e_{12}, e_{21}, e_{22}$ between (34) and (36)

Secondly, we are considering quasisynchronization.

Example 2 Consider the drive inertial memristive neural network 
where

$$
\left\{\begin{aligned}
\dot{p}_{1}(t)= & -p_{1}(t)+q_{1}(t), \\
\dot{p}_{2}(t)= & -p_{2}(t)+q_{2}(t), \\
\dot{q}_{1}(t)= & \left(-1+d_{1}-c_{1}\right) p_{1}(t)+\left(1-d_{1}\right) q_{1}(t) \\
& +a_{11}\left(p_{1}(t)\right) f_{1}\left(p_{1}(t)\right) \\
& +a_{12}\left(p_{1}(t)\right) f_{2}\left(p_{2}(t)\right) \\
& +b_{11}\left(p_{1}(t)\right) f_{1}\left(p_{1}(t-\tau(t))\right) \\
& +b_{12}\left(p_{1}(t)\right) f_{2}\left(p_{2}(t-\tau(t))\right)+I_{1}, \\
\dot{q}_{2}(t)= & \left.-1+d_{2}-c_{2}\right) p_{2}(t)+\left(1-d_{2}\right) q_{2}(t) \\
& +a_{21}\left(p_{2}(t)\right) f_{1}\left(p_{1}(t)\right) \\
& +a_{22}\left(p_{2}(t)\right) f_{2}\left(p_{2}(t)\right) \\
& +b_{21}\left(p_{2}(t)\right) f_{1}\left(p_{1}(t-\tau(t))\right) \\
& +b_{22}\left(p_{2}(t)\right) f_{2}\left(p_{2}(t-\tau(t))\right)+I_{2},
\end{aligned}\right.
$$

the memristive connection weight are as follows

$a_{11}\left(p_{1}\right)=\left\{\begin{array}{c}1,\left|p_{1}\right| \leq 1, \\ -1,\left|p_{1}\right|>1,\end{array} \quad a_{12}\left(p_{1}\right)=\left\{\begin{array}{c}2,\left|p_{1}\right| \leq 1, \\ -2,\left|p_{1}\right|>1,\end{array}\right.\right.$ $a_{11}^{*}\left(y_{1}\right)=\left\{\begin{array}{c}1,\left|y_{1}\right| \leq 1, \\ -1,\left|y_{1}\right|>1,\end{array} \quad a_{12}^{*}\left(y_{1}\right)=\left\{\begin{array}{c}2,\left|y_{1}\right| \leq 1, \\ -1,\left|y_{1}\right|>1,\end{array}\right.\right.$ $a_{21}^{*}\left(y_{2}\right)=\left\{\begin{array}{c}1,\left|y_{2}\right| \leq 1, \\ -1,\left|y_{2}\right|>1,\end{array} \quad a_{22}^{*}\left(y_{2}\right)=\left\{\begin{array}{c}1,\left|y_{1}\right| \leq 1, \\ -2,\left|y_{1}\right|>1,\end{array}\right.\right.$ $b_{11}^{*}\left(y_{1}\right)=\left\{\begin{array}{c}1,\left|y_{1}\right| \leq 1, \\ -2,\left|y_{1}\right|>1,\end{array} \quad b_{12}^{*}\left(y_{1}\right)=\left\{\begin{array}{c}-1,\left|y_{1}\right| \leq 1, \\ 2,\left|y_{1}\right|>1,\end{array}\right.\right.$ $b_{21}^{*}\left(y_{2}\right)=\left\{\begin{array}{l}2,\left|y_{2}\right| \leq 1, \\ 1,\left|y_{2}\right|>1,\end{array} \quad b_{22}^{*}\left(y_{2}\right)=\left\{\begin{array}{r}2,\left|y_{2}\right| \leq 1, \\ -1,\left|y_{2}\right|>1 .\end{array}\right.\right.$

Consider the case that $p=1$. Choose $\delta=13, c_{1}=$ $1, c_{2}=-1, d_{1}=2, d_{2}=1, \tau(t)=1$. Let activation functions be $f_{1}(x)=f_{2}(x)=\sin x$, external inputs are $I_{1}=I_{2}=0$. According to Assumption 4.1 and $4.2, l^{1}=1, M_{1}=2$. When the control gains are chosen as $k_{11}=23, k_{12}=k_{21}=20, k_{22}=21$, $a_{21}\left(p_{2}\right)=\left\{\begin{array}{c}1,\left|p_{2}\right| \leq 1, \\ -1,\left|p_{2}\right|>1,\end{array} \quad a_{22}\left(p_{2}\right)=\left\{\begin{array}{c}2,\left|p_{2}\right| \leq 1, \quad-\delta<0, \text { the conditions of Theorem } 5 \text { are sat- } \\ -2,\left|p_{2}\right|>1, \text { isfied. In this case, } \gamma^{*}=\sup _{t \geq 0}\left\{M_{1}\left(\|\Delta A\|_{1}+\right.\right.\end{array}\right.\right.$ $b_{11}\left(p_{1}\right)=\left\{\begin{array}{c}1,\left|p_{1}\right| \leq 1, \\ -2,\left|p_{1}\right|>1,\end{array} \quad b_{12}\left(p_{1}\right)=\left\{\begin{array}{c}1,\left|p_{1}\right| \leq 1, \\ 2,\left|p_{1}\right|>1,\end{array}\right.\right.$ $b_{21}\left(p_{2}\right)=\left\{\begin{array}{l}2,\left|p_{2}\right| \leq 1, \\ 1,\left|p_{2}\right|>1,\end{array} \quad b_{22}\left(p_{2}\right)=\left\{\begin{array}{r}2,\left|p_{2}\right| \leq 1 \\ -1,\left|p_{2}\right|>1,\end{array}\right.\right.$

the response system with unmatched parameters is

$$
\left\{\begin{aligned}
\dot{y}_{1}(t)= & -y_{1}(t)+z_{1}(t)-k_{11} e_{11}(t), \\
\dot{y}_{2}(t)= & -y_{2}(t)+z_{2}(t)-k_{12} e_{12}(t), \\
\dot{z}_{1}(t)= & \left(-1+d_{1}-c_{1}\right) y_{1}(t)+\left(1-d_{1}\right) z_{1}(t) \\
& +a_{11}^{*}\left(y_{1}(t)\right) f_{1}\left(y_{1}(t)\right)+a_{12}^{*}\left(y_{1}(t)\right) f_{2}\left(y_{2}(t)\right) \\
& +b_{11}^{*}\left(y_{1}(t)\right) f_{1}\left(y_{1}(t-\tau(t))\right) \\
& +b_{12}^{*}\left(y_{1}(t)\right) f_{2}\left(y_{2}(t-\tau(t))\right) \\
& +I_{1}-k_{21} e_{21}(t) \\
\dot{z}_{2}(t)= & \left.-1+d_{2}-c_{2}\right) y_{2}(t)+\left(1-d_{2}\right) z_{2}(t) \\
& +a_{21}^{*}\left(y_{2}(t)\right) f_{1}\left(y_{1}(t)\right)+a_{22}^{*}\left(y_{2}(t)\right) f_{2}\left(y_{2}(t)\right) \\
& +b_{21}^{*}\left(y_{2}(t)\right) f_{1}\left(y_{1}(t-\tau(t))\right) \\
& +b_{22}^{*}\left(y_{2}(t)\right) f_{2}\left(y_{2}(t-\tau(t))\right) \\
& +I_{2}-k_{22} e_{22}(t)
\end{aligned}\right.
$$

$\left.\left.\|\Delta B\|_{1}\right)\right\}=26<\infty, M=\left\{e(t) \in R^{n} \mid\|e(t)\|_{1} \leq\right.$ $\left.\gamma^{*} / \delta\right\}=\left\{e(t) \in R^{n} \mid\|e(t)\|_{1} \leq 2\right\}$. The initial values are given as $p_{1}(t)=2.5, p_{2}(t)=3.3, q_{1}(t)=$ $2, q_{2}(t)=-1, y_{1}(t)=-0.5, y_{2}(t)=-0.8, z_{1}(t)=$ $0, z_{2}(t)=2.5, t \in[-1,0]$. The simulation results are shown by Fig. 2-6, it can be seen that the norm of error trajectories converge into the region $M=\left\{e(t) \in R^{n} \mid\|e(t)\|_{1} \leq 2\right\}$, which verify the effectiveness of Theorem 5 .

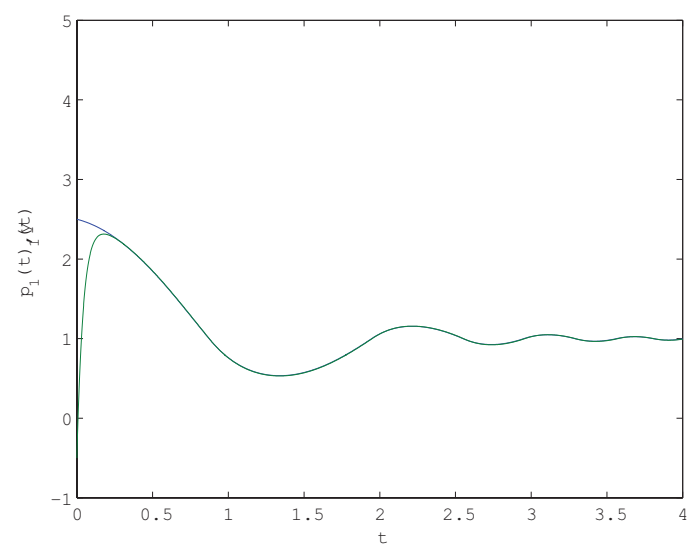

Figure 2. State trajectories of $p_{1}(t)$ and $y_{1}(t)$ 


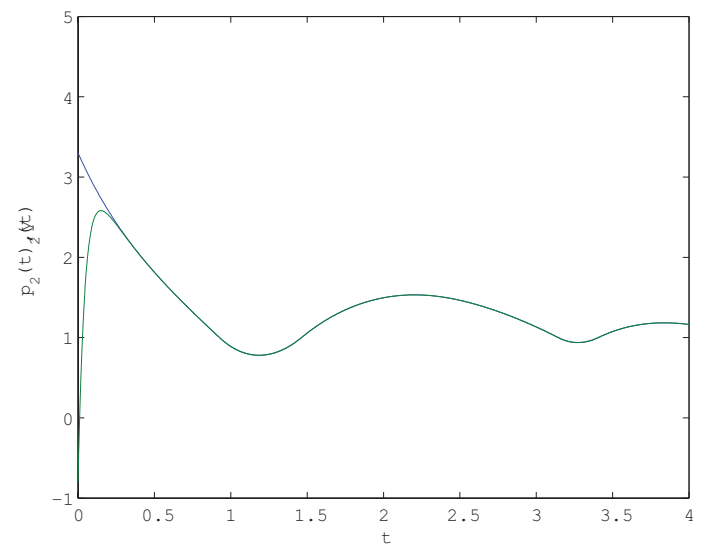

Figure 3. State trajectories of $p_{2}(t)$ and $y_{2}(t)$

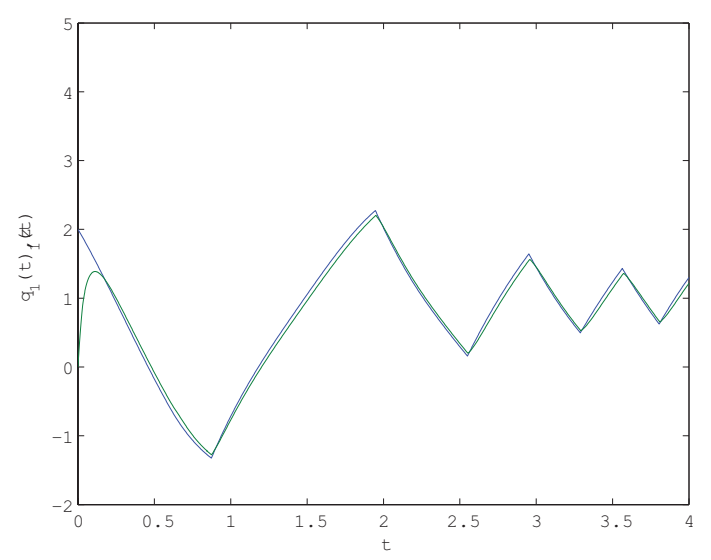

Figure 4. State trajectories of $q_{1}(t)$ and $z_{1}(t)$

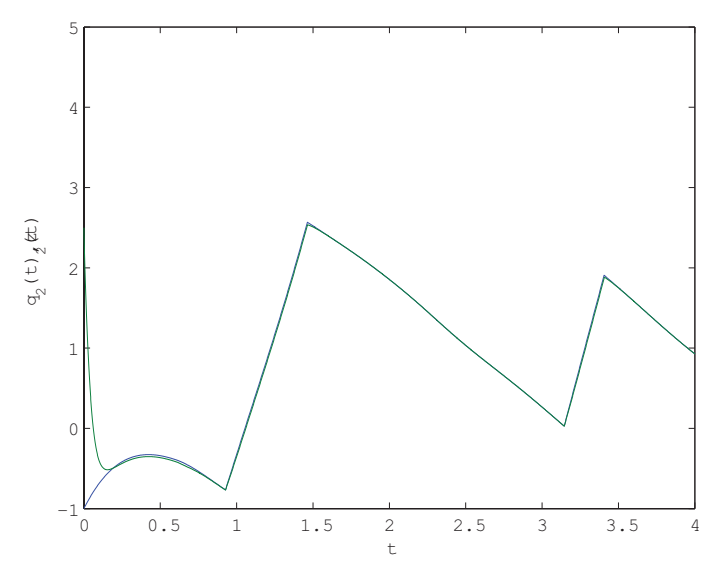

Figure 5. State trajectories of $q_{2}(t)$ and $z_{2}(t)$

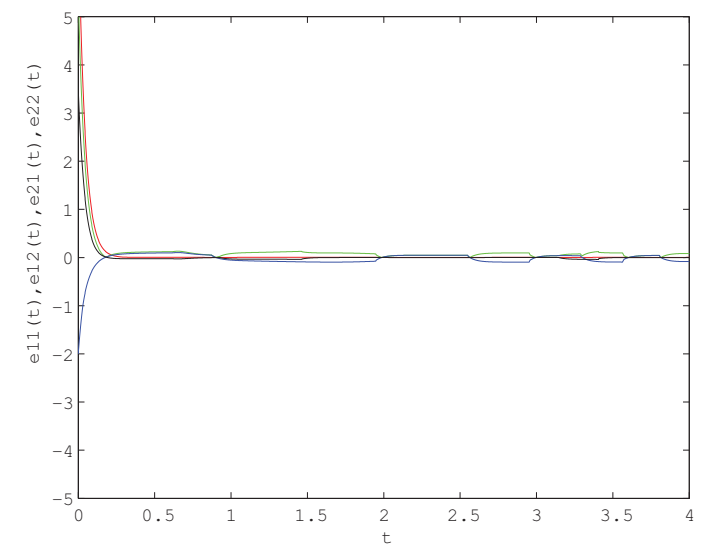

Figure 6. Synchronization error $e_{11}, e_{12}, e_{21}, e_{22}$ between (37) and (39)

\section{Conclusion}

In this paper, exponential synchronization and quasi-synchronization are studied respectively for the inertial memristive neural networks with and without parameter mismatch. Firstly, by using the Fillipov discontinuous theory and a variable transformation, the problem of second-order equations can be converted into first-order one. Then, state feedback controller and adaptive controller is proposed to study exponential synchronization, two sufficient conditions are derived. By constructing a simple controller and using matrix method measure, criterion for quasi-synchronization of the parameter mismatch case is obtained. Finally, numerical simulations are given to verify the effectiveness of our theoretical results.

Future research efforts will be devoted towards addressing the following aspects: 1) Considering the finite-time synchronization of inertial memristive neural networks via event-triggered scheme. 2) Introducing the stochastic elements into the synchronization problem of inertial memristive neural networks and trying to find impulsive control method for this issue.

\section{References}

[1] L. O. Chua, Memristor-the missing circuit element, IEEE Transaction on Circuit Theory, Vol. 18, No. 
6, pp. 507-519, 1971.

[2] D. Strukov, G. Snide and D. Stewart, The missing memristor found, Nature, Vol. 453, No. 6, pp. 8083, 2008.

[3] R. Rakkiyappan, S. Premalatha, A. Chandrasekar and J. Cao, Stability and synchronization of innertial memristive neural networks with time delays, Cognit. Neurodyn., Vol. 10, No. 5, pp. 437-451, 2016.

[4] N. Li and J. Cao, Lag synchronization of memristor-based coupled neural networks via $\omega$ measure, IEEE Trans. Neural Netw. Learn. Syst., Vol. 27, No. 3, pp. 169-182, 2016.

[5] X. Yang, J. Cao, and J. Liang, Exponential Synchronization of memeristive neural networks with delays: Interval matrix method, IEEE Trans. Neural Netw. Learn. Syst., 10.1109/TNNLS.2016.2561298.

[6] J. Hu, J. Cao and A. Elaiw, Pinning synchronization of coupled inertial delayed neural networks, Cognit. Neurodyn., Vol. 9, No. 3, pp. 341-350, 2015.

[7] Q. Liu, X. Liao and Y. Liu, Dynamics of an inertial two-neuron system with time delay, Nonlinear Dyn., Vol. 58, No. 3, pp. 573-609, 2009.

[8] Z. Zhang and Z. Quan, Global exponential stability via inequality technique for inertial BAM neural networks with time delays, Neurocomputing., Vol. 151, No. 3, pp. 1316-1326, 2015.

[9] Q. Liu, X. Liao and Y. Wu, Stability of bifurcating periodic solution for a single delayed inertial neuron model under periodic excitation, Nonlinear Analysis: Real World Applications, Vol. 10, No. 4, pp. 2384-2395, 2009.

[10] W. D. Wheeler and W. C. Schieve, Stability and Chaos in an inertial two-neuron system, Physica B, Vol. 105, No. 4, pp. 267-284, 1997.

[11] Y. Ke and C. Miao, Stability and existence of periodic solutions in inertial BAM neural networks with time delay, Neural Computing and Applications, Vol. 23, No. 3, pp. 1089-1099, 2013.

[12] W. Zhang, C. Li and T. Huang, Exponential stability of inertial BAM neural networks with timevarying delay via periodically intermittent control, Neural Computing and Applications, Vol. 26, No. 7, pp. 1781-1787, 2015.

[13] Y. Ke and C. Miao, Stability analysis of inertial Cohen-Grossberg-type neural networks with time delays, Neurocomputing., Vol. 117, No. 6, pp. 196205, 2013.
[14] J. Qi, C. Li and T. Huang, Stability of delayed memristive neural networks with time-varying impulses, Cognit. Neurodyn., Vol. 8, No. 5, pp. 429436, 2014.

[15] R. Rakkiyappan, G. Velmurugan and J. Cao, Stability analysis of memristor-based fractional-order neural networks with different memductance functions, Cognit. Neurodyn., Vol. 9, No. 2, pp. 145 $177,2015$.

[16] L. Chen, R. Wu and J. Cao, Stability and synchronization of memristor-based fractional-order delayed neural networks, Neural Netw., Vol. 71, pp. 37-44, 2015.

[17] A. Wu and Z. Zeng, Global Mittag-Leffler stabilization of fractional-order memristive neural networks, IEEE Trans. Neural Netw. Learn. Syst., Vol. 3, pp. 1-12, 2015.

[18] W. Wang, L. Li and Y.Yang, Synchronization control of memristor-based recurrent neural networks with perturbations, Neural Netw., Vol. 53, pp. 8-14, 2014.

[19] X. Yang, J. Cao and W. Yu, Exponential synchronization of memristive Cohen-Grossberg neural networks with mixed delays, Cognit. Neurodyn., Vol. 8, pp. 239-249, 2014.

[20] Y. Wan, J. Cao and W. Yu, Robust fixed-time synchronization of delayed Cohen-Grossburg neural networks, Neural Netw., Vol. 73, pp. 86-94, 2016.

[21] J. Cao and J. Lu, "Adaptive synchronization of neural networks with or without time-varying delays, Chaos, Vol. 16, No. 1, pp. 8-14, 2006.

[22] J. Cao and Y. Wan, Matrix measure strategies for stability and synchronization of inertial neural network with time-delays, Neural Netw., Vol. 53, pp. 165-172, 2014.

[23] W. He and J. Cao, Exponential synchronization of chaotic neural networks:a matrix measure approach, Nonlinear Dyn., Vol. 55, pp. 55-65, 2009.

[24] H. Bao and J. Cao, Finite-time generalized synchronization of nonidentical delayed chaotic sysytems, Nonlinear Analysis: Modelling and Control, Vol. 21, No. 3, pp. 306-324, 2016.

[25] L. Wen, Y. Yu and W. Wang, Generalized Halanay inequalities for dissipativity of Voterra functional differential equations, Journal of Mathematical Analysis and Applications Vol. 347, No. 1, pp. 169-178, 2008.

[26] M. Vidyasagar, Nonlinear System Analysis, Prentice Hall, Englewood Cliffs, 1993. 
[27] A. F. Filippov and F. M. Arscott, Differential equations with discontinuous right hand sides: control systems, Dordrecht, Kluwer, Springer, 2013.
[28] L. Xu and X. Wang, Mathematical Analysis Method and Examples, Higher Education Press, Beijing, 1983.

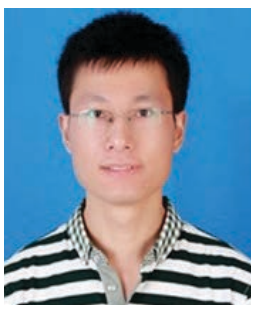

Ruoyu Wei received the M.S. degree in Applied Mathematics from Southeast University, Nanjing, China in 2017. He is currently pursuing the PH.D. degree with Southeast University, Nanjing, China. His current research interests include neural networks, multiagent systems and control theory.

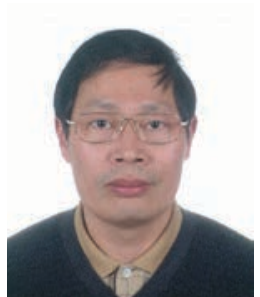

Jinde Cao is an Endowed Chair Professor, the Dean of School of Mathematics and the Director of the Research Center for Complex Systems and Network Sciences at Southeast University. From March 1989 to May 2000, he was with the Yunnan University. In May 2000, he joined the School of Mathematics, Southeast University, Nanjing, China. From July 2001 to June 2002, he was a Postdoctoral Research Fellow at Chinese University of Hong Kong, Hong Kong. Professor Cao was an Associate Editor of the IEEE Transactions on Neural Networks, and Neurocomputing. He is an Associate Editor of the IEEE Transactions on Cybernetics, IEEE Transactions on Cognitive and Developmental Systems, Journal of the Franklin Institute, Mathematics and Computers in Simulation, Cognitive Neurodynamics, and Neural Networks. He is a Fellow of IEEE, a Member of the Academy of Europe, a Member of European Academy of Sciences and Arts, and a Foreign Fellow of Pakistan Academy of Sciences. He has been named as Highly-Cited Researcher in Engineering, Computer Science, and Mathematics by Thomson Reuters/Clarivate Analytics. He received the National Innovation Award of China (2017). 